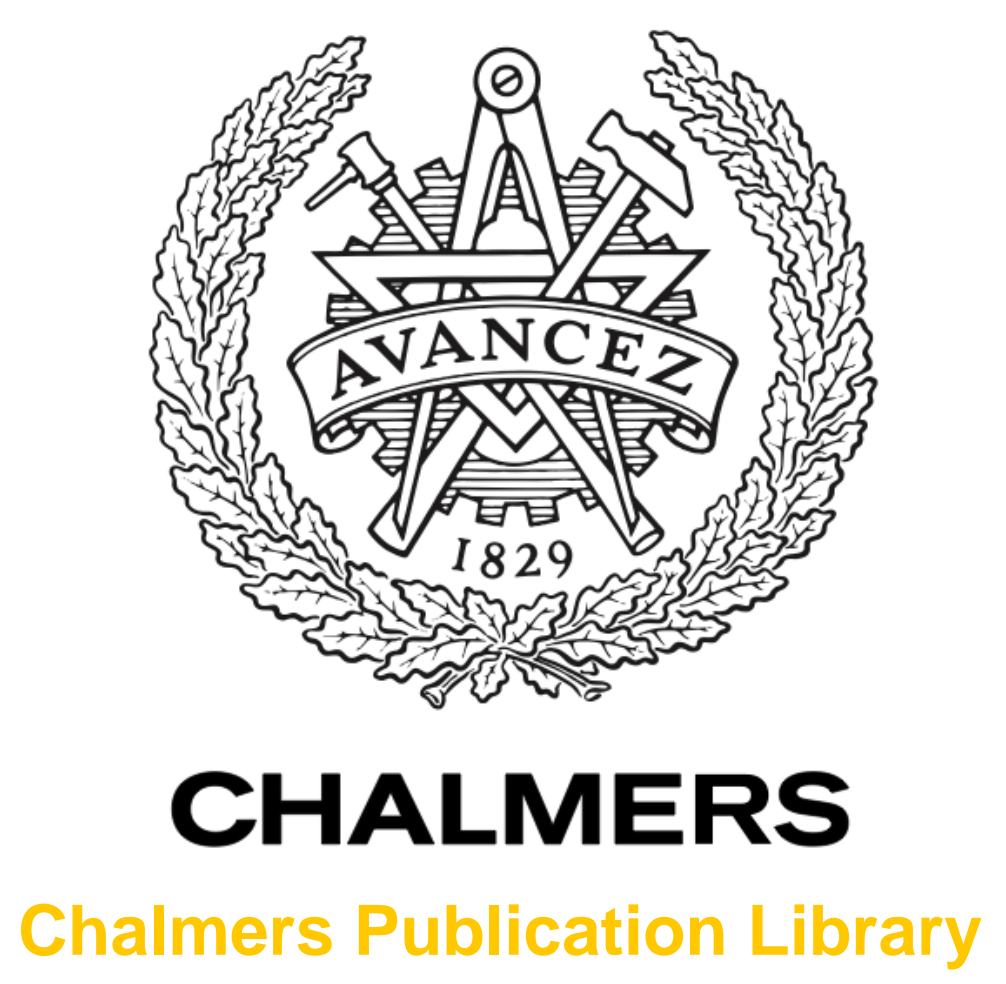

\title{
Terahertz GaAs Schottky diode mixer and multiplier MIC's based on e-beam technology
}

This document has been downloaded from Chalmers Publication Library (CPL). It is the author's version of a work that was accepted for publication in:

Indium Phosphide and Related Materials (IPRM), 2013 International Conference (ISSN:

1092-8669)

Citation for the published paper:

Drakinskiy, V. ; Sobis, P. ; Zhao, H. (2013) "Terahertz GaAs Schottky diode mixer and multiplier MIC's based on e-beam technology". Indium Phosphide and Related Materials

(IPRM), 2013 International Conference

http://dx.doi.org/10.1109/ICIPRM.2013.6562606

Downloaded from: http://publications.lib.chalmers.se/publication/182857

Notice: Changes introduced as a result of publishing processes such as copy-editing and formatting may not be reflected in this document. For a definitive version of this work, please refer to the published source. Please note that access to the published version might require a subscription.

Chalmers Publication Library (CPL) offers the possibility of retrieving research publications produced at Chalmers University of Technology. It covers all types of publications: articles, dissertations, licentiate theses, masters theses, conference papers, reports etc. Since 2006 it is the official tool for Chalmers official publication statistics. To ensure that Chalmers research results are disseminated as widely as possible, an Open Access Policy has been adopted.

The CPL service is administrated and maintained by Chalmers Library. 


\title{
Terahertz GaAs Schottky diode mixer and multiplier MIC's based on e-beam technology
}

\author{
V. Drakinskiy ${ }^{\text {1a }}$, P. Sobis ${ }^{2}$, H. Zhao ${ }^{1}$, T. Bryllert ${ }^{1,3}$, and J. Stake ${ }^{1}$ \\ ${ }^{1}$ Terahertz and Millimetre Wave Laboratory, Department of Microtechnology and Nanoscience, \\ Chalmers University of Technology, SE-412 96 Gothenburg, Sweden \\ ${ }^{2}$ Omnisys Instruments AB, SE-43132 V. Frölunda, Sweden \\ ${ }^{3}$ Wasa Millimeter Wave AB, Gothenburg, Sweden \\ avladimir.drakinskiy@chalmers.se
}

\begin{abstract}
We present the progress of the technological development of a full e-beam based monolithically integrated Schottky diode process applicable for sub-millimetre wave multipliers and mixers. Evaluation of the process has been done in a number of demonstrators showing state-of-the-art performance, including various multiplier circuits up to $200 \mathrm{GHz}$ with a measured flange efficiency of above $35 \%$, as well as heterodyne receiver front-end modules operating at $340 \mathrm{GHz}$ and $557 \mathrm{GHz}$ with a measured receiver DSB noise temperature of below $700 \mathrm{~K}$ and $1300 \mathrm{~K}$ respectively.
\end{abstract}

Keywords - Schottky diodes, passive circuits, membrane, submillimeter wave mixers, multipliers

\section{INTRODUCTION}

There is a need for efficient and reliable heterodyne receivers operating in the sub-millimetre wave band above 300 $\mathrm{GHz}$ for future space science missions and earth observation instruments. The sub-millimetre wave regime allows the study of different meteorological phenomena such as water vapour, the ice and water content in clouds, and ice particle sizes and distribution, which are important parameters for the hydrological cycle of the climate system and the energy budget of the atmosphere.

Today Schottky diode mixers and multipliers are the key elements for millimetre and sub-millimetre wave roomtemperature heterodyne receiver systems. At frequencies up to around $400 \mathrm{GHz}$ discrete diode technology can be applied. The planar Schottky diode topology has proven reliable and is today used in most commercial mixer and multiplier circuits.

At higher frequencies $(>400 \mathrm{GHz})$ monolithic integration is needed due to better fabrication and alignment tolerances as well as to enable more advanced circuit integration. Moreover, the performance and functionality of discrete diode circuit designs, is limited by the shape and thickness of the supporting substrate. One of the solutions is the fabrication of monolithic integrated circuits (MICs) supported by a thin membrane. Numerous results based on this technique have been reported [1]. In this paper we present a full e-beam based Terahertz MIC GaAs Schottky membrane process enabling advanced circuit integration well up in the $\mathrm{THz}$ range.

\section{DIODE FABRICATION}

\section{A. Schottky diode mixer on membrane}

The Chalmers diode process is based on electron beam lithography, with a beam spot of less than $5 \mathrm{~nm}$, allowing precise and repeatable anode and air bridge formation. Hence, this process module can also be utilized for submicron size anodes and terahertz monolithic integrated circuits (TMICs). Scanning Electron Microscope images of a mixer diode is shown in Figure 1.

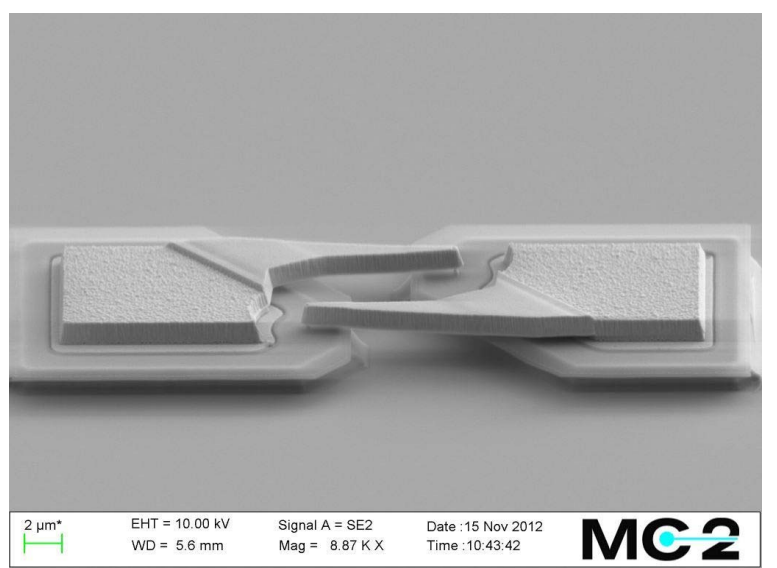

Figure 1. SEM image of an antiparallel diode with an anode area of $0.1 \mu \mathrm{m}^{2}$ designed for operating at $1.2 \mathrm{THz}$ and fabricated at MC2 Chalmers.

For the diodes on membrane, the starting structure is a semi-insulating GaAs substrate supporting a $3 \mu \mathrm{m}$ thick GaAs layer sandwiched in between two AlGaAs etch stop layers and a buffer and an active layers. The standard diode fabrication process is as follows:

- Deposition of a stress-balanced PECVD $\mathrm{SiO}_{2}$ layer.

- Patterning of the ohmic contacts, wet etching through the $\mathrm{SiO}_{2}$ layer and the active layer of GaAs and deposition of the ohmic contacts metallization with following lift off process.

- Annealing of the ohmic contacts.

- Patterning of the Schottky contacts, wet etching through the $\mathrm{SiO}_{2}$ layer and deposition of the Schottky contacts metallization with following lift off process.

- Patterning of the air bridge and deposition of metallization with following lift off process.

- Isolation of the diode by wet etching.

- Patterning of the membrane shape, wet etching of $3 \mu \mathrm{m}$ GaAs using a selective etchant, which stops etching on the bottom AlGaAs layer.

- Patterning of passive circuitry e.g. beamleads, waveguide probes and filter structures and deposition of metallization with following lift off process. 
- Thinning down the sample from the backside to the AlGaAs layer which is then etched away to release the devices.

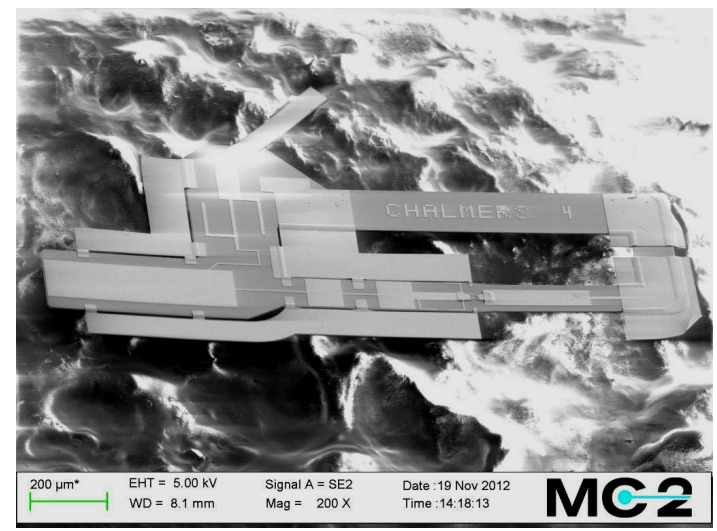

Figure 2. SEM image of a released monolithically integrated Schottky membrane mixer designed for operating at $557 \mathrm{GHz}$ and fabricated at $\mathrm{MC} 2$ Chalmers.

In Figure 2, a $557 \mathrm{GHz}$ membrane mixer MIC developed under the TeraComp FP7 EU project is shown. With this particular mixer design an optimum receiver noise level of less then 1300 $\mathrm{K}$ DSB including all losses has been reached with several assembled mixer modules, using an external IF LNA with a $\mathrm{T}_{\min }$ of $30 \mathrm{~K}$. The result by itself is a redefinition of state of the art performance for room temperature receivers at these frequencies, but also an important indication for the device quality of our process [2].

\section{B. Schottky doublers on membrane}

The device technology described above can also be used for GaAs Schottky multipliers. Results for our narrowband GaAs Schottky varactor multipliers show a very good agreement between model simulations and measurements indicating good process control.

In Figure 3 a Schottky membrane doubler mounted in a waveguide block module is shown. The design is optimized for a high efficiency and low input power. The measurements show state-of-the-art results that are presented in Figure 4.

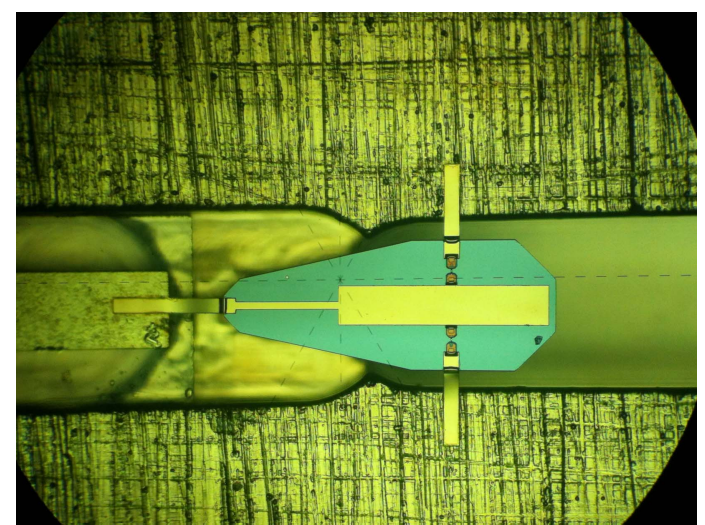

Figure 3. Photograph of an assembled Schottky membrane doubler, designed for an operating output frequency of $170 \mathrm{GHz}$ and fabricated at MC2 Chalmers.

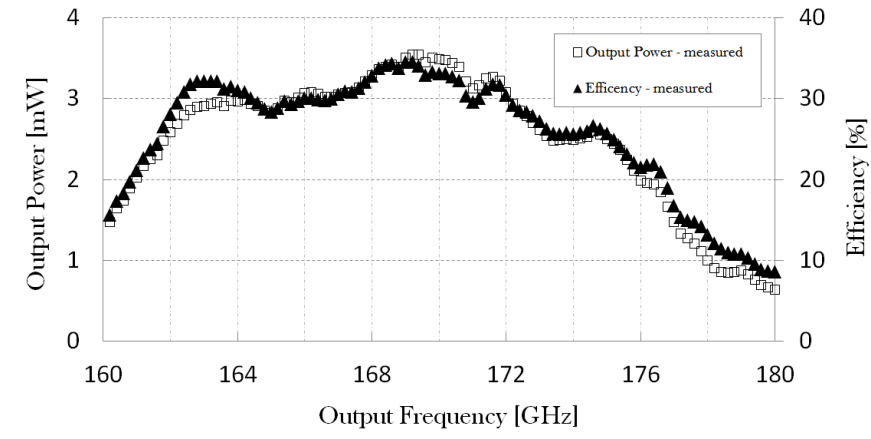

Figure 4. Measured results for a low power $170 \mathrm{GHz}$ high efficiency Schottky membrane doubler, with a measured bandwidth of nearly $10 \%$ and with a $3.5 \mathrm{~mW}$ of output power running at $35 \%$ efficiency.

Figure 5 shows the measured results for a broad band Schottky doubler, which covers a full waveguide band with more than $10 \%$ conversion efficiency and good return-loss.

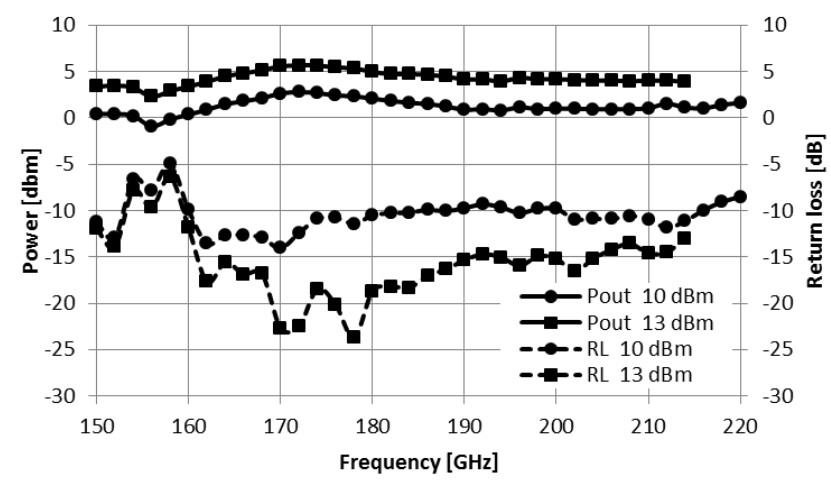

Figure 5. Measured results for a broadband Schottky membrane doubler covering a full waveguide band.

\section{CONCLUSIONS}

The fabrication process of monolithically integrated Schottky diode mixers and multipliers for THz applications has been developed. The agreement between the results and simulations indicates good control and stability of the process.

\section{ACKNOWLEDGMENT}

The work was supported by the EU FP7 project "TERACOMP" under grant № 242424. The work was also carried out in the GigaHertz Centre, Gothenburg, Sweden.

\section{REFERENCES}

[1] P.H. Siegel, R.P. Smith, S. Martin, and M. Gaidis, "2.5 THz GaAs monolithic membrane-diode mixer," IEEE Trans. Microw. Theory Tech., vol.47, no. 5, pp. 596-604, May 1999.

[2] H. Zhao, V. Drakinskiy, P. Sobis, J. Hanning, T. Bryllert, A.-Y. Tang, and J. Stake, "Development of a $557 \mathrm{GHz}$ GaAs monolitic membranediode mixer", 24th International Conference on Indium Phosphide and Related Materials, Audust 2012. 